\title{
Quality of Experience enforcement in wireless networks ${ }^{\star}$
}

\author{
R. Serral-Gracià, M. Yannuzzi, E. Marin-Tordera, X. Masip-Bruin, S. Sánchez \\ \{rserral, yannuzzi, eva, xmasip, sergio\}eac.upc.edu
}

Advanced Network Architectures Lab, Technical University of Catalunya (UPC), Spain

\begin{abstract}
In this paper we present a Multimedia Wireless Management System, which can be used on-line to assess and guarantee the quality of multimedia traffic in a wireless network. The proposed platform uses both network and application layer metrics to build up a scalable quality assessment of multimedia traffic. Moreover, the system provides traffic provisioning capabilities by coordinating the network access and usage both from the wireless node and from the network access point. These two combined features permit our platform to guarantee a satisfactory multimedia user experience in wireless environments. We evaluate our proposal by issuing an experimental deployment in a testbed and performing a series of tests under different network situations to demonstrate the Quality of Experience guaranties of our system. The results show that the quality of video perceived by end-users is considerably improved compared to the typical wireless network.
\end{abstract}

Key words: QoE, Wireless Network Management, Multi-layer

\section{Introduction}

The massive market penetration of new mobile devices such as laptops, mobile phones, and netbooks, all with different Internet connection capabilities, encouraged the broad deployment and adoption of wireless technologies such as GPRS, Bluetooth, or Wireless LAN (WLAN) everywhere. In addition, the good performance/cost tradeoff of the latter favored the apparition of a vast number of hotspots offering Internet connectivity, e.g., at the city's airport, at the university campus, or even at the coffee shop next door, contributing to increase the user's effective on-line periods [1].

This "always connected" attitude acquired by the users has greatly affected the network usage patterns. Indeed, watching on-line TV programs, video-chatting with family or friends, or visiting video-streaming sites are now common practices. As a consequence, all this new multimedia traffic has introduced a series of network constraints in terms of latency and data transmission reliability, that network technologies must comply with in order to offer a competitive network connection. Despite of this, WLAN and other mobile technologies using a shared medium access, do not have mechanisms to guarantee such reliability.

\footnotetext{
* This work was partially funded by Spanish Ministry of Science and Innovation under contract TEC2009-07041, and the Catalan Government under contract 2009 SGR1508.
} 
To overcome this limitation, in this paper we present a Multimedia Wireless Management System (MWMS), offering an on-line multimedia traffic quality monitoring and enforcing platform for wireless environments. Hence the contribution of this work is twofold:

1. First, we propose a multi-layer approach to on-line multimedia traffic quality assessment and enforcement, which combines application and networking data in order to guarantee higher priority for the multimedia flows in the wireless network.

2. And second, our solution uses a two-way approach, i.e., traffic control both from the network access point and from the end-node, to overcome the inherent unreliability of the wireless medium, hence guaranteeing the proper quality of multimedia flows.

Our system uses both network metrics such as used bandwidth and packet losses; and application metrics, namely, the Mean Opinion Score (MOS), in order to make informed management decisions. The main difference of our approach compared to other existing solutions [2] is that we use information from different layers, and combine them at both edges of the wireless network to provide a complete resource management platform.

We experimentally evaluate our proposal in our testbed by comparing the perceived user experience, i.e., Quality of Experience (QoE), between our platform and the generic case of unmanaged network resources. To do this comparison, we generate a video stream using a well-known multimedia streaming application under different network service degradation scenarios. The results show that the quality of the flows is properly maintained during the streaming when the network is managed by our MWMS, while the user perception is clearly degraded in the same conditions with an unmanaged network.

The rest of the paper is structured as follows, the next section details some related work about the requirements in order to design a QoE assessment and enforcement platform. In Sections 3 and 4 we describe our MWMS, and the policies used to perform the resource management. In Section 5, we detail the experimental testbed used and evaluate our platform. We continue in Section 6 with the analysis of the scalability of our solution, and finally in Section 7, we conclude and outline our future lines of research in this topic.

\section{Related Work}

A full-fledged Multimedia Wireless Management System must consider three important aspects in its design, $i$ ) it needs mechanisms to compute the QoE, ii) then it must consider the medium reliability, specially in wireless links, and finally iii) it requires mechanisms for resource reservation.

QoE sets the umbrella of techniques used for the subjective analysis of the end-user perception of a given service. This subjectiveness, can be made objective by means of well-known techniques, such as the MOS [3], combined with the E-Model [4] for voice transmission, or techniques such as [5] for video streams. This paper does not intend to propose a new method for computing the QoE, but rather to exploit existing techniques to receive feedback about the quality delivered to the user, so that we can dynamically 
manage the resources on the wireless network. In particular, in this work we compute the multimedia flow's QoE by using the technique detailed in [6], a solution which is able to estimate the perceived QoE from a single point of analysis by using frame loss and frame type information at the application layer.

Regarding the reliability of the wireless environment, in the case of WLAN, there are some efforts to provide Quality of Service (QoS) in the Wireless MultiMedia extensions (WMM), which in conjunction with the standardized medium access algorithm defined in 802.11e [7] can prioritize certain packets on the network. However, this standard even if based on a powerful medium access control, is insufficient to have higher level guaranties of proper multimedia quality, specially from the end-user perspective. Most of the contributions in quality assessment in wireless environments are based on low level mechanisms to provide QoS [2,8], but neither considering user's perception nor application's specific constraints. Our MWMS, specifically uses QoE metrics as the control axis for the resource management.

Similarly to our approach, in [9] the authors propose a cross-layer QoE framework for HSDPA networks, the main difference with our approach is that they center the quality improvements by re-adapting the requirements of the applications for improving the MOS, while we improve the user perceived quality by prioritizing the Multimedia traffic.

Finally, the last identified challenge in our platform is the definition of the mechanisms to prioritize the traffic in the network. In this regard, there are several existing techniques both in commercial routers as in commodity operating systems (ACLs, firewalls, etc.), which permit to limit and control the up and downstream load of the wireless network. More specifically, in our work we use [10] as a solution that provides a very versatile mechanism, namely Traffic Control, to enable fine control of the upstream by priority queuing the packets matching a specific criteria, and the downstream, e.g., by delaying TCP's ACK packets.

\section{Multimedia Wireless Management System}

The Multimedia Wireless Management System (MWMS) presented in this paper is a multi-layer approach to on-line assessing and enforcing of the QoE in multimedia flows. MWMS proposes an efficient platform that combines information from different layers and provides a very fast and reliable traffic provisioning mechanism for multimedia flows in wireless environments.

The complete framework is presented in Figure 1 . The MWMS is mainly composed of three different parts, first the Client Side, with two different tasks, to assess the user perceived QoE, and to manage the wireless link usage of the end-node, by locally detecting possible causes of service degradation. Second, the Network Side, which controls the access to the whole wireless network by relying on the multimedia flows' QoE as a metric. And third, the Server Side, which streams the media over the network. In this work we focus on the management system on the client and network sides, leaving the end-to-end QoE assessment and provisioning as an important part of our future work. 


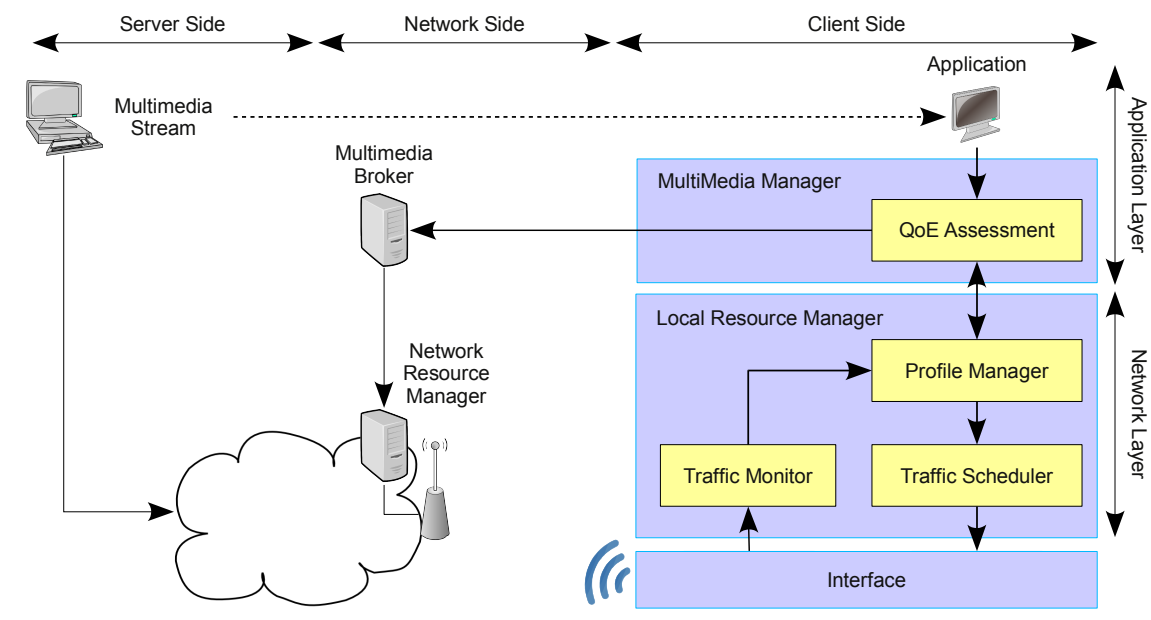

Fig. 1. The Multimedia Wireless Management System (MWMS).

\subsection{Client Side Resource Management}

QoE assessing of multimedia flows is not a trivial task, as it involves comparison of the original and received video frames using PSNR [5], which means that this mechanism cannot be used in a real-time assessment platform. However, this approach can be simplified by using approximations such as [6]. In this case, the best location to perform the user satisfaction assessment is within the destination multimedia application, where the most accurate information about the data delivered to the user can be gathered. To this end, we propose to directly adapt the end-user application in order to infer the delivered QoE, while giving feedback both to the Local Resource Manager (cross-layer vertical signaling) and to the Multimedia Broker (horizontal signaling). It is worth noticing that the application's adaptation only involves to inform the MWMS about the lost frames and their type in the video stream [6].

Local Resource Manager: The Local Resource Manager (LRM) performs the low level monitoring and measurement tasks of the wireless link in the local node. LRM is composed by three different entities, i.e., the Traffic Monitor, the Profile Manager and the Traffic Scheduler.

The Traffic Monitor monitors in real-time the bandwidth and resource usage of the wireless node, periodically reporting its status to the Profile Manager. Then the Profile Manager computes the amount of raw resources required in terms of bandwidth, delay, jitter, and packet losses in order to optimize the resource reservation performed by the Traffic Scheduler. Such parameters are inferred from the effective requirements imposed by the Multimedia Manager. Finally, the Traffic Scheduler prioritizes the multimedia traffic over the rest of the wireless node traffic in case of service degradation. 
MultiMedia Manager: The MultiMedia Manager (MMM) gathers quality information directly from the client application, it issues periodic queries about the experienced QoE, and receives information about the resource availability in the wireless node from the lower layers. The task of the MMM is twofold. On the one hand, if the service degradation is caused by other applications within the wireless node, the Multimedia Manager instructs the LRM to increase the priority of the multimedia flows. On the other hand, if the degradation is due to the wireless network, the MMM requests more resources to the Multimedia Broker on the Network Side.

\subsection{Network Side Resource Management}

Given the shared medium nature of wireless links, in order to provide service guaranties and a reliable resource allocation mechanism, the network must be controlled from both sides of the link. The Network Side Resource Management manages the resources in the downstream to the wireless node, and it is composed of two different parts, i.e., the Multimedia Broker and the Network Resource Manager.

Multimedia Broker: The Multimedia Broker (MB) is the entity within the network that receives the feedback from all the subscribed multimedia applications about the experienced user satisfaction in real-time. The MB interfaces with the Network Resource Manager in order to instruct whether more resources are needed, and as we detail in the next section, it bases the resource reservation decision depending on a preset acceptable QoE boundary. By design, the MB is independent of the underlying network, consequently it does not consider specific network metrics, but it only triggers resource reservations to the Network Resource Manager.

Network Resource Manager: The Network Resource Manager (NRM), analogously to the LRM, is responsible of guaranteeing that the multimedia traffic is received properly by the end-user. To this end, the NRM defines the different traffic profiles recognized by the system, and maps the input received by the MB to actual network parameters. Then, at the lower layer, it also controls the overall access to the wireless environment by scheduling the traffic within the wireless access point. With this twoway approach (i.e., client and network sides), the system can effectively guarantee the offered service to the user from both sides of the wireless network.

One important task of the network side resource management left as future work is the connection management and prioritization among the multimedia flows. This can be accomplished by a Connection Access Control (CAC) system, by introducing a business model with billing and accounting processes to define user categories, or by a combination of both alternatives.

\section{Quality of Experience Assessment and Enforcement}

After the introduction of the MWMS building blocks, this section is devoted to the description of the specific criteria used for reserving and releasing the mentioned resources. As outlined previously, MWMS defines two modes of resource allocation, local node resource management and network resource management. 


\subsection{Local resource management}

The local resource management is driven by two different layers, first the low level resource allocation and second the high level QoE assessment.

The low level local resource allocation policy is based on the local's node resource usage monitoring and reservation as follows:

Definition 1. Let $\mathcal{U}_{t} \in[0,1]$ be the used resources at time $t$ in the input queue for the wireless node. Then $\mathcal{U}_{t}$ :

$$
\mathcal{U}_{t}=R_{t}+\mathcal{B}_{t}
$$

Where $R_{t}=\sum_{j=1}^{n} r_{j}$, with $R_{t} \in[0,1]$, are the ratio of resources needed for all the flows $r_{1 . . n}$ under surveillance by the system, and $\mathcal{B}_{t}$ is the usage of the rest of the incoming background traffic toward the node at time $t$. Then, if $\mathcal{U}_{t}>\Theta$, where $\Theta$ is the upper bound for a reliable wireless link usage, the system issues a reservation as instructed by the Profile Manager for the $R_{t}$ resources. It is important to notice that $R_{t}$ is easy to compute because the resource usage of multimedia flows is rather constant over time.

Similarly to the resource reservation, the resources are released when the link usage drops below $\theta$, i.e., $\mathcal{U}_{t}<\theta$, where $\theta$ is the lower safety bound in the link usage to trigger the resource release, or when the multimedia flow finishes, as advertized by MMM. The pseudo-code to implement the whole LRM can be found in Algorithm 1 . It is worth noticing, that measuring the link usage is a straight-forward task because it only involves to query the queue status of the interface, as provided by kernel data structures.

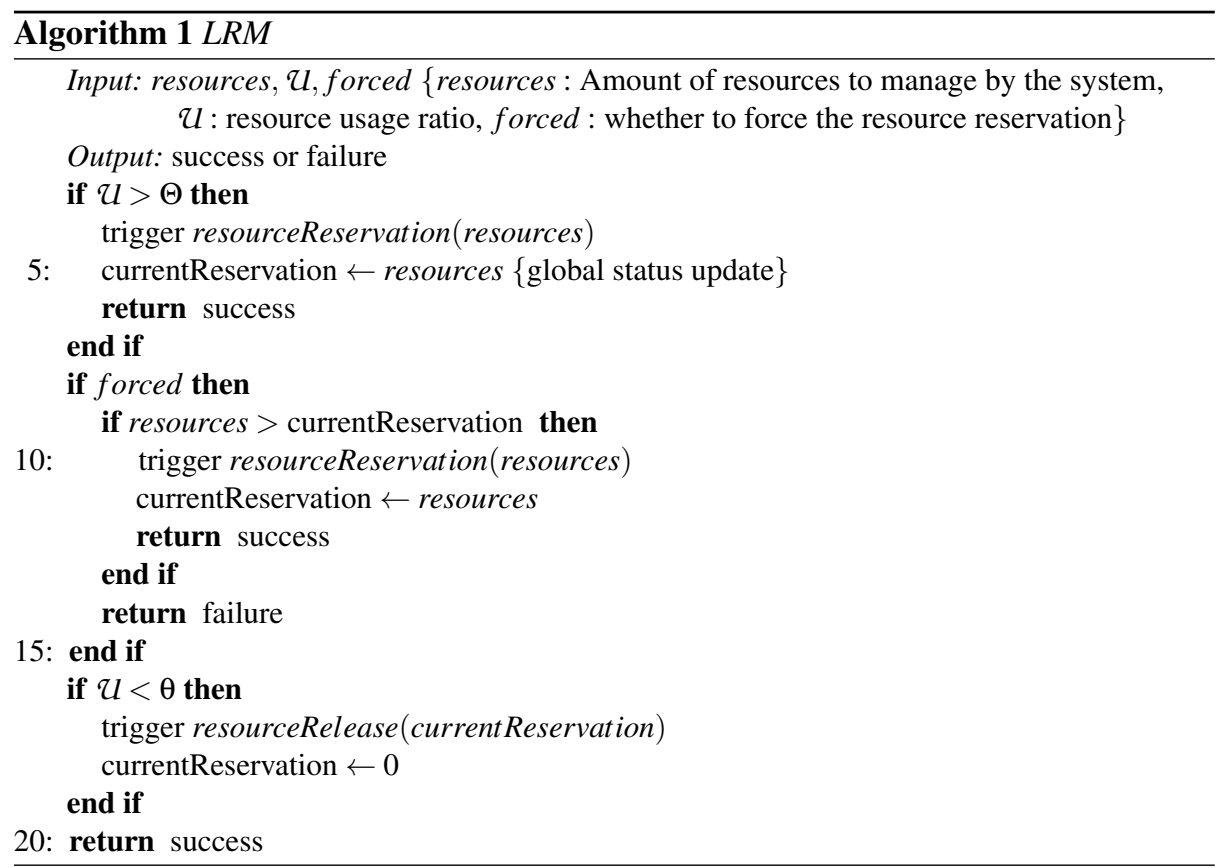


To complement the LRM, both allocation and release of resources can also be triggered by the higher MMM layer. Hence, besides the strict low level resource usage, the system must also consider the user perception of the delivered multimedia traffic. Therefore, the MMM assesses the QoE of the received multimedia streams as described below (we refer interested readers to [6] for a throughout description of the MMM QoE assessment).

Let $\sigma \in[1,5]$ be the lower tolerable MOS threshold for a multimedia flow. Then, for any multimedia flow $j$ under surveillance present on the system, if the quality of experience $q(t)$ at time $t$ of any flow $j$ is $q_{j}(t) \leq \sigma$, MMM will trigger a local resource reservation request (vertical multi-layer signaling) to the LRM. If the reservation is not successful, or if the quality continues below $\sigma$, despite the reservation, MMM will trigger a network resource reservation request (horizontal signaling). The pseudo-code implementing the MMM process is detailed in Algorithm 2 .

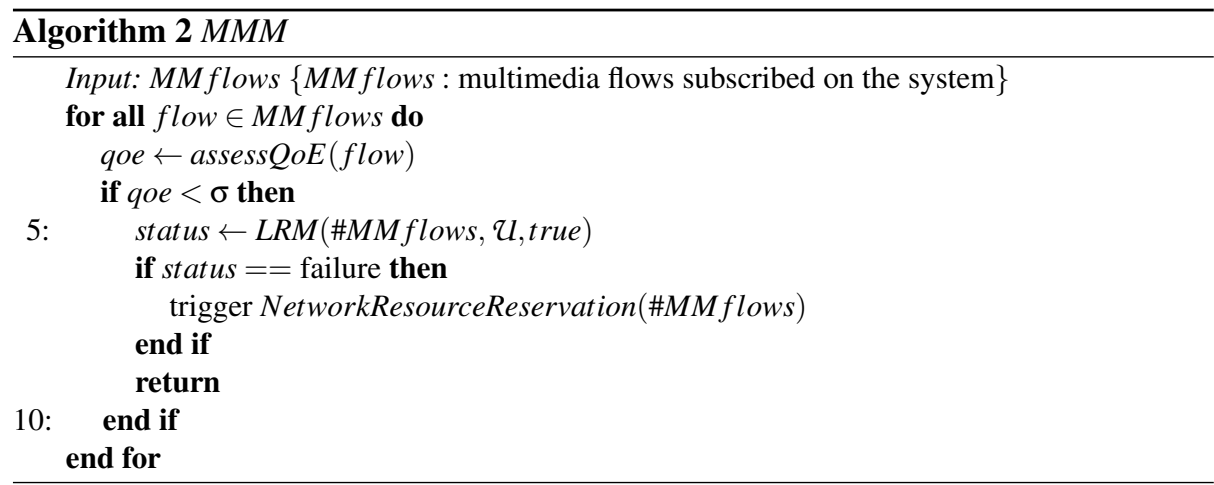

As it can be noted from Algorithms 1 and 2, the LRM keeps track of the current status of the reservation and reports whether the reservation is successful or not. This process can be invoked in two different ways, periodically, as a traffic monitor, or it can be invoked by the MMM in case the QoE is below $\sigma$ (see line 5 in Algorithm2).

\subsection{Network resource management}

Analogously to the local resource management, the network resource management is composed of two different parts, the low level resource reservation, performed by the NRM and the high level management performed by the MB. The interfaces between these two levels are similar to the one detailed in the LRM and the MMM (as detailed in Algorithms 1 and 2). However, a key difference is the fact that the MB needs to consider all the reservation requests of all the nodes in the wireless network, opposed to the single node approach of the local resource management. Analogously the NRM will prioritize the multimedia flows subscribed into the system towards all the wireless nodes. 
The flow subscription is issued by the MMM, which gathers the properties of the flow and informs the MB about specific application constraints, in terms of frame losses and required bandwidth.

\section{Experimental Evaluation}

This section details the tests, the testbed used, and the evaluations performed to validate the MWMS. All the evaluations are focused on comparing the end-user's MOS obtained by running MWMS against a typical unmanaged network.

\subsection{Tests and testbed}

The testbed used to perform the MWMS validation is illustrated in Figure 2 It is composed by the Multimedia Streaming Server (MSS), the Network Access Point (NAP) and the Wireless Node (WN). All three components are standard PC using the Linux Debian Operating system with traffic control capabilities to manage the resources. In the testbed the MB and the NRM are embedded within the NAP, while in the WN we set up the MMM and the LRM, together with the video streaming client. On its side, the video server is configured to stream using the RealTime Protocol (RTP), a high quality vided ${ }^{1}$ of $1024 \times 576$ pixels with a bit rate of $5.5 \mathrm{Mbps}$ for both audio and video. The streaming application used is VideoLan Client (VLC) [11].

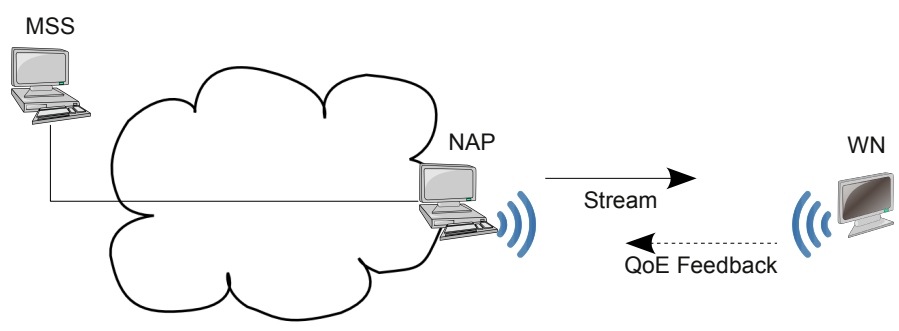

Fig. 2. Testbed main blocks

In order to test the MWMS we deployed our system as follows. We set up a high delay variation queue with limited capacity in order to produce a random amount of packet losses in the wireless network. In the WN we monitored the MOS of the traffic as described in Section 4, with a $\sigma=3$ as advised in [5], $\Theta=0.8$ and $\theta=0.5$, and with an update interval of 1 second. Once the violation of QoE is assessed, the NRM configures a priority queue for the multimedia flows.

With the above methodology we simulate three different service degradation levels: low, medium and high. Finally, we run the same set of tests disabling our management system to compare the results in the same conditions.

\footnotetext{
1 The Elephant's Dream - http://www.elephantsdream.org
} 


\subsection{MWMS evaluation}

The experimental evaluation of the proposed system is performed by running the tests specified previously. In our first study, we verify that the MWMS is able to provide better QoE during the whole test than the case with unmanaged network resources. To this end, in Figure 3 we show the Cumulative Density Function (CDF) of the obtained MOS over time, in the left we show the results for MWMS and in the right the case without any network management. For the sake of clarity, we transposed the CDFs, this way the X-Axis shows the normalized fraction of time where the quality of the video stream is below $q_{j}(t)$ (for all $t$ in the test), while in the Y-Axis shows the instantaneous MOS for each test.

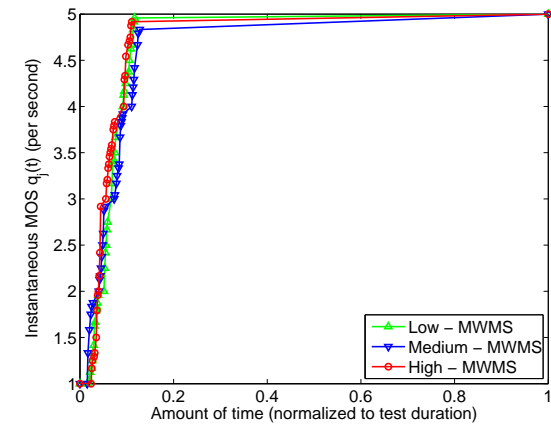

(a) MWMS

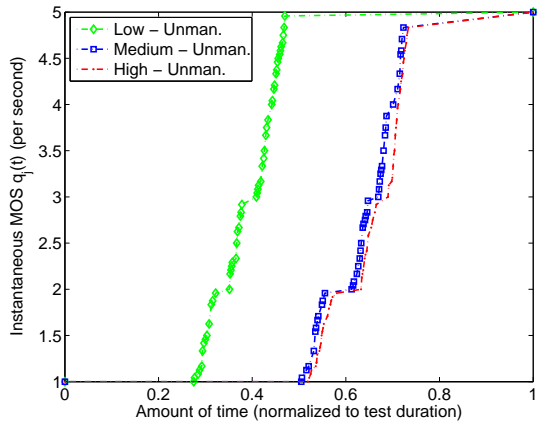

(b) Unmanaged

Fig. 3. Transposed Cumulative Density Function of the MOS per degradation level

The figure highlights that MWMS easily outperforms the unmanaged case for all the tests, and independently of the level of service degradation the quality is very good most of the time. In particular we can see that in the case of MWMS only $\sim 6 \%$ of the time the obtained MOS is below 3, while in the case of an unmanaged network it ranges from $\sim 35 \%$ to $\sim 65 \%$ of the time, i.e., around half of the streaming duration. Moreover, when MWMS is not present, between 30 and $50 \%$ of the time the quality reaches the lowest possible MOS value, i.e., 1, meaning that the video and audio are completely disrupted. Opposed to this, in the case of MWMS this value is around $1 \%$, which roughly corresponds to the assessment and QoE enforcement setup time of the system.

It is worth noticing that the results for high and medium service degradation levels, the unmanaged network obtain similar results. The reason for that is the exponential degradation of the service in the presence of frame losses.

In order to analyze in more detail these results, in Table 1 we detail the numeric values in terms of quality. In the first column, we show the average MOS obtained for each one of the tests. As it can be noted, the quality is constant, around 4.75, when using MWMS, while it degrades in the case of an unmanaged network. In the second column we computed the $5^{\text {th }}$ Percentile, which identifies the lower bounds in quality for the tests. As before, managing the system mostly avoids having low quality periods 
given the relatively high value of the percentile. The third and fourth columns in the table complement the above information by indicating the amount of service degradation periods, and the percentage of time with a degraded system, respectively. In particular, we can see that the degradation periods are fairly constant over the tests for the managed system, while they are much bigger when disabling the management. Since each period is equivalent to 1 second of streaming, we can notice that the amount of service degradation intervals are between $\sim 8$ and $\sim 11$ times higher when not using MWMS. Analogously, we can observe that in the worst case the percentage of time with service degradation with high degradation level is kept at $4.4 \%$ with MWMS, while it is $\sim 15$ times worse without our management solution.

\begin{tabular}{|c||l|l|l|l|}
\hline & Average & $5^{\text {th }}$ Prc. & \# Degr & $\%$ Degr. \\
\hline \hline Low MWMS & 4.74 & 2 & 38 & $5.9 \%$ \\
Low Unmanaged & 3.45 & 1 & 243 & $37.8 \%$ \\
Medium MWMS & 4.73 & 2.78 & 35 & $5.4 \%$ \\
Medium Unmanaged & 2.44 & 1 & 409 & $64.7 \%$ \\
High MWMS & 4.76 & 3 & 28 & $4.4 \%$ \\
High Unmanaged & 2.38 & 1 & 427 & $66.5 \%$ \\
\hline
\end{tabular}

Table 1. Evaluation results: Columns 1 and 2 show MOS values for the different tests, Columns 3 and 4 the degradation periods

In the results it can be noted that the MOS of the high degradation level with MWMS is better than the rest. This is caused by the fact that with high service degradation MWMS is able to react faster as the MOS decreases in less time and the degradation is more noticeable, activating the resource provisioning faster.

Another interesting study is the analysis of the duration of continuous service degradation periods. In Figure 4 we show the maximum and the average continuous duration of the service degradation in the network for each test, the left figure shows the results for MWMS, while the case without management can be found on the right figure. As it can be observed, the least degradation period with MWMS is of 15 seconds, while it goes up to 94 seconds without MWMS. Regarding the average values, they are below five seconds with MWMS, raising up between 8 and 18 for the unmanaged system.

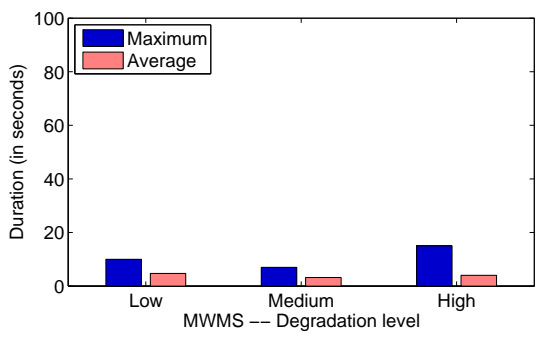

(a) MWMS

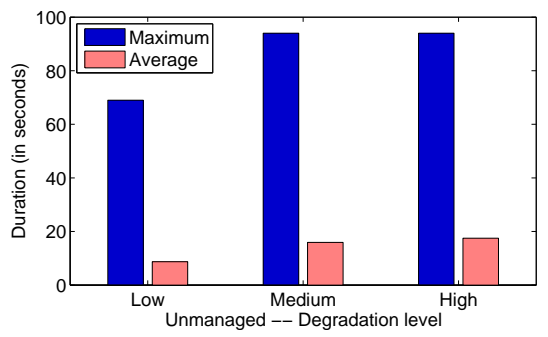

(b) Unmanaged

Fig. 4. Maximum and average continuous periods of service degradation 
To finish our study, we further analyzed the causes of the degradation periods when using MWMS, and we observed that they were caused by external interferences of other nearby wireless networks, which cause burst of losses which are uncontrollable by our platform. However, even with these side effects, we claim that our system outperforms and delivers much better performance than an unmanaged network with a very simple yet effective platform.

\section{Discussion}

The final concern of any quality assessment and enforcement system is whether it is sufficiently scalable to be usable in a real deployment. In our case, MWMS is easily scalable given its inherently distributed design. In particular, each wireless node is in charge of locally monitoring the resources used, which only involves simple packet counting, and computing of instantaneous local bandwidth usage, while the application layer analyzes lost frames, which indeed is part of the multimedia decoding algorithm, and thus it is already performed on the system.

On the other hand, in the networking side, the NAP does not need to monitor the status of the network, since its functionality is limited to allocate resources when instructed. Such resource reservation is straight-forward because it only involves simple queue management, which is already present in most operating system's networking stack.

Added to the above, a wireless network has tight constraints in terms of available bandwidth and medium access; imposing hard limits in the maximum possible number of multimedia flows that can be served simultaneously. Therefore, depending on the used codec for the streaming and the required per flow bandwidth, a wireless system with $54 \mathrm{Mbps}$ can manage a very limited amount of simultaneous flows, e.g. less than 50 assuming $1 \mathrm{Mbps}$ streams ${ }^{2}$, which is easily manageable with current queuing algorithms.

Finally, in terms of the bandwidth required to exchange the QoE information with the NAP, in MWMS the only feedback to the system is issued when a disruption is found, and given that the resources are released once the load of the system is reduced or the streaming has finished, it permits the system to greatly optimize the amount of required traffic, which is negligible compared with the existing network traffic.

\section{Conclusions and Future Work}

In this paper, we presented a Multimedia Wireless Management System (MWMS) which performs two different tasks. On the one hand, it is able to accurately assess the QoE of multimedia flows directly from application's data, and on the other hand, it guarantees the multimedia traffic quality by using low level resource management mechanisms. In addition, by directly acting over the access point of the network, the system is allowed to have a very tight control over the incoming traffic to the wireless

\footnotetext{
${ }^{2}$ Given the overhead and the performance reduction caused by the MAC algorithms.
} 
network. This together with the QoE based feedback given from the wireless node, makes our MWMS a competitive solution for wireless network resource provisioning.

Given the versatility of our solution, we were able to evaluate its performance in a real environment under a different set of conditions, which prove that MWMS provides an effective QoE assessment and enforcement mechanism for multimedia streaming over wireless networks. Moreover, given the distributed and lightweight design of MWMS, the system can easily scale for managing a wireless network environment.

As an important part of our future work, one complementary part to complete the platform is the implementation of an admission control system in the wireless network in order to make a more fair use of the resources for all the nodes. This is to avoid the potential starvation of non-multimedia flows or the excess of resource reservation by some nodes in the wireless network,.

Another line open for further research, is to develop an end-to-end mechanism, which combined with this local approach can guarantee the multimedia quality even in the case that the cause of the service degradation is not in the end-user network.

Finally, we plan to upgrade our platform by integrating support for the Wireless Multimedia extensions present in 802.11e, which can further improve the quality of multimedia flows.

\section{References}

1. Rideout, V.J., Foehr, U.G., Roberts, D.F.: GENERATION $M^{2}$ Media in the Lives of 8- to 18-Year-Olds. A Kaiser Family Foundation Study (Jan 2010)

2. Lee, J., Liao, W., Chen, J.M., Lee, H.H.: A practical QoS solution to voice over IP in IEEE 802.11 WLANs. IEEE Communications Magazine 47(4) (2009) 111-117

3. ITU-T Recommendation G.113: Transmission impairments due to speech processing $(02 / 2001)$

4. ITU-T Recommendation G.107: The E-model, a computational model for use in transmission planning $(03 / 2005)$

5. Klaue, J., Rathke, B., Wolisz, A.: EvalVid - A Framework for Video Transmission and Quality Evaluation. In: In Proceedings of the 13th International Conference on Modelling Techniques and Tools for Computer Performance Evaluation. (2003) 255-272

6. Serral-Gracià, R., et. al: Packet Loss based Quality of Experience of multimedia video flows. In: R. Rep.http://personals.ac.upc.edu/rserral/research/techreports/ psnr_mos.pdf (2009)

7. IEEE Computer Society, IEEE Std 802.11e: Part 11: Wireless LAN Medium Access Control (MAC) and Physical Layer (PHY) specifications Amendment 8: Medium Access Control (MAC) Quality of Service Enhancements (Nov 2005)

8. Hock, D., Bayer, N., Pries, R., Siebert, M., Staehle, D., Rakocevic, V., Xu, B.: Qos provisioning in WLAN mesh networks using dynamic bandwidth control. In: European Wireless 2008, Prague, Czech Republic (June 2008)

9. Thakolsri, S., Khan, S., Steinbach, E., Kellerer, W.: QoE-Driven Cross-Layer Optimization for High Speed Downlink Packet Access. Journal of Communications 4(9) (2009) 669

10. : Linux Advanced Routing and Traffic Control -http://lartc.org/

11. : Videolan media player -http://www.videolan.org/vlc 